\title{
Le doctorat en architecture
}

Un procès au réel

\section{Thierry Verdier}

\section{(2) OpenEdition}

Journals

Édition électronique

URL : http://journals.openedition.org/crau/372

DOI : $10.4000 /$ crau. 372

ISSN : 2547-5746

Éditeur

Éditions du patrimoine

\section{Édition imprimée}

Date de publication : 1 décembre 2014

Pagination : 47-56

ISBN : 978-2-7577-0379-3

ISSN : 1296-4077

Référence électronique

Thierry Verdier, « Le doctorat en architecture », Les Cahiers de la recherche architecturale et urbaine [En ligne], 30/31 | 2014, mis en ligne le 14 septembre 2017, consulté le 10 décembre 2020. URL : http:// journals.openedition.org/crau/372 ; DOI : https://doi.org/10.4000/crau.372 


\section{Le doctorat en architecture \\ Un procès au réel}

Le doctorat en architecture existe. La réforme des études d'architecture en France, avec l'application des rythmes pédagogiques issus de la déclaration de Bologne et ses cycles d'études divisés entre licence, master et doctorat, a fait émerger du néant réglementaire le vocable de "doctorat en architecture ». Que celui-ci ait existé par le passé, dans d'autres pays ou sous d'autres latitudes, était certainement présent à l'esprit des législateurs. Mais par-delà l'apparition d'un grade académique choisi pour enrichir le vocabulaire d'une formation supérieure en architecture, ce fut bien de reconnaissance universitaire dont il fut question. Souffrant d'une sorte de timidité vis à vis des vieilles institutions que sont les universités, les écoles d'architecture françaises n'osaient à peine affirmer leur existence scientifique. Pourtant quarante années de recherches, de production scientifique, de prix et d'excellence savante, auraient dû totalement effacer cette gêne qui marquait la petite école contrainte de rivaliser avec ses grandes aînées de l'enseignement supérieur. Aujourd'hui la question ne se pose plus, et les inscriptions en doctorat en architecture se multiplient. 
Cependant, l'architecture, par l'infini des savoirs et des compétences qu'elle représente, ne peut se circonscrire dans le cadre étroit d'une discipline académique ou universitaire ${ }^{1}$. Peut-on admettre que le doctorat en architecture soit contraint de se soumettre aux grands cadres scientifiques et étanches distinguant les disciplines universitaires entre elles? Certainement pas. Tout a été mis en place pour faire passer l'architecture au tamis de ses savoirs multiples. L'architecture n'est pas une discipline au sens universitaire du terme, mais une confluence indéfinie de savoirs que l'on se doit de clairement distinguer les uns des autres ${ }^{2}$. Revendiquant sa dette vis-à-vis de l'histoire, apportant quelques pincées de sociologie à son approche des établissements humains, multipliant les études urbaines au sens de l'aménagement opérationnel, inscrivant sa logique constructive dans la doxa du développement durable, enrichissant son corpus technique par la maîtrise des avancées notables en représentation numérique ou paramétrique, en thermique passive, en pensée constructive, etc., la pensée architecturale s'est épanouie dans l'allégresse de son universalité. Or, dans un univers de production scientifique codifié par les règles étroites de la lisibilité thématique, l'architecture se trouve bien à l'étroit dans un costume dessiné par d'autres, et à d'autres fins.

Des masses de travaux (intellectuels, techniques, théoriques ou pratiques) sont pourtant venus enrichir la culture architecturale française. On ne compte plus les études pionnières qui ont orienté l'évolution de la pensée de la ville ou de l'histoire de l'architecture, qui ont orienté aussi la compréhension des savoir-faire et des processus de conception, qui ont orienté enfin une certaine manière de penser l'art d'édifier par-delà les questions de style, de décor ou d'emballage ornemental. Interrogeant la technique comme un savoir et non comme un savoirfaire, radicalisant la réflexion historique sous I'angle du projet, transportant le jeu d'acteurs de la commande dans le monde de l'intention, considérant le désastre environnemental sous l'angle de la dissolution du lien social autant que territorial, etc., les chercheurs français en architecture ont inventé leurs propres outils et fait émerger de nouveaux concepts. En dresser l'inventaire serait impossible et relèverait de "l'insensé » (au sens étymologique du mot) tant cette recherche fut plurielle, nomade et souvent braconnière.

Mais il est une réalité qui transcende l'idée même d'une définition si amphibologique soit-elle, et cette réalité a longtemps était écrite sous la forme du mot "projet». On verra plus loin ce qu'il faut entendre aujourd'hui de ce vocable-tiroir. Or, ce terme porte en lui l'idée d'un devenir, d'un a-tendu, ou d'une projection vers l'inconnu d'une chose à naître. Et c'est autour de cette notion que pourraient se regrouper tous les travaux qui s'inscrivent dans cette case malléable qui s'appelle le doctorat en architecture. Pour l'heure, il importe plutôt d'en faire l'apologie critique, à défaut d'en fabriquer l'exégèse.

\section{Une culture de la critique}

L'idée de modernité, projet inachevé selon Jürgen Habermas $^{3}$, définit une démarche intellectuelle qui s'est ouverte avec I'humanisme de la Renaissance et qui depuis, a traversé tous les débats théoriques ${ }^{4}$. Postmodernité et bientôt posthumanisme ${ }^{5}$ sont apparus,
1. Cet article est issu de l'expérience du doctorat en architecture sur travaux développée à l'université Paul-Valéry - Montpellier 3 (école doctorale 58, «Langues, littératures, cultures, civilisations »/EA 4424 Centre de recherches interdisciplinaires en sciences humaines et sociales de Montpellier). On pourra, à cet égard, relire avec intérêt les différentes communications et positions présentées dans: Vers un doctorat en architecture, recherche architecturale, urbaine et paysagère (sous la direction d'Éric Lengereau), Paris, ministère de la Culture et de la Communication, 2005. Pour compléter ces regards et ces analyses, voir: Architecture et construction des savoirs: quelle recherche doctorale? (sous la direction d'Éric Lengereau), Paris, Éditions Recherches/ministère de la Culture et de la Communication, 2008.

2. Benoît Goetz, Chris Younès, Philippe Madec, L'indéfinition de l'architecture, Paris,
Éditions de la Villette, coll. "Passage», 2009, p. 8-19.

3. Jürgen Habermas, «La modernité un projet inachevé ", Critique, t. 37, no 413, octobre 1981, p. 950-969. II n'est pas neutre que Paul Chemetov et Jean-Claude Garcias aient repris cette formule comme titre de leur (contre) exposition (La Modernité, un projet inachevé, catalogue d'exposition, Paris, Éditions du Moniteur, 1982). 
ou apparaîtront sans doute, comme les expressions ultimes de cette pensée du dépassement ${ }^{6}$. L'architecture en est l'expression durable. Elle est ce qui demeure des civilisations et des sociétés et, à cet égard, ne peut être perçue comme la simple manifestation d'un état de la technique, d'une demande sociale univoque ou d'un engouement passager pour le Beau. Pour forcer le trait et accepter d'être caricatural, il faudrait accepter l'idée que la pensée moderne est celle qui permet à I'entendement, à l'être humain (dans sa faiblesse et sa complexité) de croire en la possibilité de maîtriser une part de sa destinée. En ce sens l'architecture n'est pas la simple transformation de l'abri originel'7, elle est cette spéculation (intellectuelle, savante et technologique) qui permet, temporairement, de répondre à l'exigence de "l'être-là ${ }^{8}$ ». Le doctorat en architecture devrait avoir cette perspective à l'horizon de sa pensée.

La question est de savoir si l'on peut interroger le savoir en dépassant la question du faire. Les écoles d'architecture enseignent, entre autres, des savoir-faire. Ceux-ci sont installés dans une perspective critique, mais demeurent avant tout les éléments qui permettent la constitution d'un bagage professionnel. Les écoles d'architectures sont des écoles professionnelles dans le sens où elles forment à un métier. Mais elles sont aussi plus que cela. On y apprend la différence entre « faire de I'architecture » et « être architecte ». Pendant longtemps, l'enseignement de l'architecture s'était organisé dans la transmission des manières, des bases théoriques et techniques, et des modèles d'excellence. La pseudorupture de mai 1968 - n'en déplaise aux nostalgiques d'une société libre et libérée - n'a pas radicalement changé cet état de fait. Sans doute certains axiomes sociologiques et comportementaux sont venus enrichir la panoplie des savoir-faire. Mais la "culture d'atelier", avec son lot de corrections subjectives et d'énoncés péremptoires, avec sa logique de la référence admirable et ses coups de crayon autoritaires, n'existe que par la quête d'une preuve ontologique autoproclamée. II est certes incontestable que l'enseignement dans les écoles d'architecture françaises s'est considérablement amélioré. Mais cela tient davantage de l'enrichissement du corpus de références admissibles (pourrait-il en être autrement dans un monde où l'échange et le virtuel ont rétréci l'échelle d'appréhension de l'infini?) que d'une métamorphose radicale des modes pédagogiques. Après tout, qu'importe si les modèles d'enseignement n'ont pas

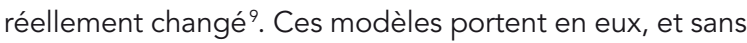
doute malgré eux, le caractère même de la conception en architecture: son inconstance doctrinaire, sa confrontation entre réel imaginé et imagination réalisable, et surtout cette immense complexité de formalisation qui résiste encore aux travaux les plus pointus en sciences cognitives. L'architecture se construit ainsi dans une culture de la critique opportune ${ }^{10}$, avec des fondements scientifiques parfois contestables, mais particulièrement opérants dans une dynamique de conception.

$C^{\prime}$ est pourquoi, la distinction entre une critique qui ne serait que l'expression d'un goût subjectif et la théorie qui serait l'apothéose d'une véritable démarche heuristique, n'a qu'une valeur bien ténue dans un univers où la création doit primer. Mais qui dit création, ne dit pas déferlement incohérent de savoirs approximatifs et de formes échevelées. La théorie architecturale s'élabore dans une
4. Voir Lawrence E. Cahoone, The Dilemma of Modernity. Philosophy, Culture, and Anti-Culture, Albany (NY), State University of New York Press, 1987, qui dresse un large spectre de cette évolution depuis la Renaissance, René Descartes et Emmanuel Kant jusqu'à Donald Winnicott, Ernst

Cassirer et John Dewey.

5. Voir Jean-Michel Besnier, Demain les posthumains. Le futur a-t-il encore besoin de nous? Paris, Fayard, 2010. Gilles
Lipovetsky, Jean Serroy, L'esthétisation du monde. Vivre à l'âge du capitalisme artiste, Paris, Gallimard, 2013.

6. Jean-Louis Cohen, assisté de Vanessa Grossman, La modernité, promesse ou menace? France. 101 bâtiments 1914-2014, Éditions Dominique Carré/Institut français, 2014. Ce catalogue d'exposition pour le Pavillon français de la Biennale d'architecture de Venise 2014 en dresse un bilan, au final, assez sévère.
7. Joseph Rykwert, On Adam's House in Paradise: The Idea of the Primitive Hut in Architectural History, Greenwich (CT), New York Graphic Society, 1972, (trad. fr. : La Maison d'Adam au paradis: l'idée de la hutte primitive dans l'histoire architecturale, Paris, Le Seuil, 1976), p. 151-154.

8. Olivier Chadoin, Être architecte, les vertus de l'indétermination. De la sociologie d'une profession à la sociologie du travail professionnel, Limoges, Presses 
démarche de " critique en direct » ${ }^{11}$ qui certes, n'est pas la garantie de son universalité, mais qui offre l'avantage d'une remise en cause et d'une interrogation perpétuelle. $C^{\prime}$ est donc bien à partir de cet habitus de classe ${ }^{12}$ que peut se percevoir la première caractéristique du doctorat en architecture. Cela demande à être théorisé, mis en perspective scientifique et certainement interrogé "de l'intérieur ». Mais cela permet tout au moins de croire que "le projet», tel que l'entendent les architectes, correspond à ce moment de la conception qui voit émerger un objet dans le déploiement d'une formalisation provisoire - l'objet n'étant pas nécessairement une chose définie formellement, et la formalisation n'usant pas nécessairement des cadres traditionnels de la représentation. Le " projet " est donc cette culture de la critique qui peut se circonscrire dans une démarche itérative capable de fédérer des informations complexes et parfois même contradictoires. II s'agit d'une manière de penser, non d'un objet identifiable. II s'agit d'une démarche, d'une posture, non d'une expression ${ }^{13}$. La démarche d'un tout qui dirait l'œuvre n'est sans doute pas crédible. Mais elle présente l'avantage de mettre en place des dispositifs ${ }^{14}$ intellectuels qui sont précisément la base de l'enseignement du projet en école d'architecture.

Or, I'architecte qui s'engage dans une recherche doctorale ne doit pas oublier d'où il vient. II n'a pas à rompre avec la tradition de sa formation et la pertinence de son métier. S'engager dans une recherche qui I'amènerait à utiliser (maladroitement) les outils d'une autre discipline conduirait à l'à-peu-près, au bricolage, certainement pas à l'efficience scientifique. Demandet-on à un chirurgien qui fait une thèse d'utiliser le langage de la linguistique, à un juriste d'user des concepts de la physique quantique, à un musicien de devenir un expert en biologie? Pourtant lorsque l'on regarde les sujets de doctorats en architecture qui émergent depuis quelques années, il n'est pas toujours possible de mesurer en quoi telle ou telle recherche relève de l'architecture et non de I'histoire de l'architecture, du génie civil, de la résistance des matériaux, de l'informatique ou des sciences de I'ingénieur. II faut donc aller au-delà du sujet, au-delà de l'intitulé du doctorat, pour comprendre comment le corpus d'étude est analysé avec les outils conceptuels propres à l'architecte. II ne faudrait pas, en effet, que le doctorat en architecture ne soit qu'une formule générique désignant toute thèse menée, plus ou moins, en école d'architecture, avec des outils qui ne seraient pas (nécessairement) ceux de la discipline architecture. Car s'il s'avérait que pour complaire aux cadres disciplinaires de l'université, les doctorats en architecture devinssent des doctorats dans d'autres disciplines que l'architecture, alors l'invention même du doctorat en architecture aurait échoué.

\section{Une culture du projet}

Quels que soient les sujets traités et les outils conceptuels convoqués pour mener une recherche, un travail d'historien se reconnaîtra toujours comme un travail d'historien. L'école des Annales a totalement modifié le territoire des historiens ${ }^{15}$. En ouvrant ses pages à des recherches entremêlant les savoirs et les concepts de la géographie historique et humaine, de la démographie, de l'étude quantitative, de l'histoire culturelle et sensible, des sociabilités, de la longue durée et de l'événement, universitaires de Limoges, 2013, p. 49.

9. Jean-Pierre Durand, La représentation du projet. Approche pratique et critique, Paris, Éditions de la Villette, 2003. Pascal Urbain, De la désillusion. Six cours d'architecture, Marseille, Les Éditions générales, Conseil d'architecture, d'urbanisme et de l'environnement des Bouches-du-Rhône, 2001.

10. Peter Zumthor, Thinking Architecture, Baden, Lars Müller Publishers, 1998.
11. Jean-Pierre Epron, dans L'École de I'Académie, 1671-1793, ou l'Institution du goût en architecture, Nancy, Centre d'études méthodologiques pour l'aménagement, 1984, avait déjà souligné cette formation du jeune architecte confronté à l'immédiat de la correction pour des périodes plus anciennes qui correspondent à la naissance de l'Académie d'architecture en France.

12. II faudrait revenir à Pierre Bourdieu, La distinction. Critique sociale du jugement, Paris, Éditions de Minuit, 1979, p. 110-121.

13. Souhaitant valoriser des démarches et non des " produits ", l'exposition Réenchanter le monde. Architecture, villes, transitions, 21 mai-6 octobre 2014, Paris, Cité de l'architecture et du patrimoine, a d'ailleurs souligné cette approche. Catalogue: Réenchanter le monde. L'architecture et la ville face aux grandes transitions (Marie-Hélène Contal dir., 
du mythe et la linguistique etc., les Annales ont imposé une autre manière de penser l'histoire. L'historien est même devenu, grâce à elles, une sorte de penseur de l'absolu de l'humanité. La puissance de l'école historique française s'est affirmée auprès de toutes les universités du monde entier. II s'agissait en fait d'une culture de la curiosité, capable de discerner derrière l'évidence d'un fait, d'une action ou d'un lieu, toute la complexité d'un temps. Certains ont pu s'émouvoir des contours doctrinaires qui naissaient de cette hégémonie historique ${ }^{16}$. Pourtant, un modèle était bien là.

La même chose devrait être possible dans le monde de l'architecture. Les exemples ne manquent pas, et il suffit de faire une petite plongée dans les productions des laboratoires de recherches des écoles d'architecture pour s'en convaincre. La qualité est partout présente. Au regard de la modestie de leur échelle, on peut même admettre, avec l'AERES, que les laboratoires des écoles sont souvent plus dynamiques que d'importantes machines universitaires. Les écoles participent pleinement de l'enrichissement de la communauté scientifique. Elles continuent même à orienter certaines inflexions de la recherche universitaire. Mais qu'en est-il des professionnels, des architectes praticiens, des maîtres d'œuvres dans cet univers de la recherche doctorale? Ils sont (très) peu nombreux. Ils semblent absents de l'histoire qui les concerne. Cela semble être une spécificité française. Lorsque l'on jette un simple regard rapide sur certaines publications "recherche" de l'université de Princeton, telle 30609017, ou encore Pamphlet Architecture ${ }^{18}$, la relation du projet architectural à la recherche apparaît comme une sorte d'évidence. Que dire des AA files ${ }^{19}$, des clips de $S A+P$ Press ${ }^{20}$, des travaux du LIA à Berlin ${ }^{21}$, etc. dont les contenus scientifiques relèvent exclusivement des processus de conception à l'œuvre dans la pensée architecturale. Cette même volonté se rencontre dans beaucoup de publications périodiques encadrées par les écoles d'architectures françaises ou par les enseignants de ces écoles ${ }^{22}$. La qualité scientifique de ces publications ne peut pas être remise en cause. Toutes les études présentées témoignent de la vigueur de la recherche en école d'architecture. Pourtant la part de la recherche relevant totalement d'une "culture de projet » demeure marginale. Cela ne signifie pas que ces travaux soient disjoints des modes spécifiques d'appréhension du monde par la culture architecturale. Bien au contraire, un architecte parle toujours la langue dans laquelle il a été construit ${ }^{23}$. Mais ce qui parfois fait défaut, c'est le transfert de cette langue dans un mode projectuel de recherche. C'est-à-dire interroger le lieu commun du faire architectural avec les armes et les concepts des savoirs architecturaux.

Parmi les lieux communs de l'architecture, le pont fait figure de poncif. Certes, construire un pont relève de la plus haute technicité. Pourtant celle-ci se superpose tout autant dans I'histoire des franchissements et des couvrements et relève donc de l'art d'édifier, que dans la métaphore du pont chez Martin Heidegger (qui détourne de la difficulté) ou encore du " pont flottant des songes » de la tradition japonaise. Le pont est une figure intellectuelle qui s'apparente ainsi davantage aux travaux sur la pensée des infrastructures ${ }^{24}$ qu'à une œuvre du génie civil. C'est cette polysémie qui devrait toujours se retrouver dans une approche architecturale de l'idée constructive ${ }^{25}$.
Aliki-Myrto Perysinaki coord.), Paris, Éditions Alternatives, Manifesto, 2014).

14. Alain Guiheux, Architecture dispositif, Marseille, Éditions Parenthèses, 2013. L'auteur a souligné les " dispositifs » que représente la pensée architecturale à l'œuvre.

15. Voir Emmanuel Le Roy Ladurie, Le Territoire de I'historien, Paris, Gallimard, 1973. On pourra regretter que cette vision large de l'enquête historique ait si peu abordé les questions liées à l'habiter comme théorie et ait négligé les travaux sur l'espace, les lieux de vie, l'architecture (autrement qu'à travers la question de l'usage).

16. Hervé Couteau-Bégarie, dans Le Phénomène "Nouvelle Histoire ». Stratégie et idéologie des nouveaux historiens, Paris, Economica, 1983, y vit une sorte de triomphe du matérialisme historique chez un groupe dominant (celui des historiens).

17. Fondée en 2001, la revue 306090 a été créée par des étudiants en architecture désireux de proposer aux étudiants et aux jeunes architectes un regard critique et renouvelé sur la profession. Cette revue est éditée aux Princeton Architectural Press. On y retrouve principalement les signatures de Emily Abruzzo et Jonathan D. Solomon auxquelles se joignent David. Lays, Gerald Bodziak, Eric Ellingsen, etc. 
Un autre lieu commun de la pensée architecturale se retrouverait par exemple dans les mots métropolisation et ville générique. Là encore, entre les nouvelles de la métropole froide et l'imaginaire « tiède » de l'imaginaire métropolitain ${ }^{27}$, les architectes nous ont entraînés vers des chemins qui échappent à l'urbanisme réglementaire pour s'épanouir dans la redécouverte d'une ville qui doit être une idée avant d'être un lieu ${ }^{28}$. La notion même de suburbanisme s'inscrit d'ailleurs dans cette dilution des grands horizons dogmatiques de la modernité 29. La ville franchisée, la ville tentaculaire des marques et des espaces de consommation, n'est pas simplement I'accomplissement d'une pensée néolibérale, elle est aussi le produit d'une invention pour laquelle les architectes de la performance ne sont peut-être pas totalement innocents ${ }^{30}$. On aura beau affirmer que la société postmoderne, dans laquelle l'immédiat de la jouissance a remplacé la morale de l'épreuve, est l'aboutissement logique de l'évolution d'une humanité (" libérale »); l'invention demeure le moteur de l'action. C'est peut-être cela qu'il faut traquer sous l'angle du projet doctoral? Aussi, sans qu'il soit nécessaire de prendre un à un ces mots qui submergent l'héritage architectural ${ }^{31}$, convient-il d'accepter qu'un doctorat en architecture soit un travail sur l'esprit des langues qui fait parler l'architecte à l'insu de lui-même. À l'évidence, cela est difficile pour un architecte engagé dans un travail sur « le réel ». Tout le monde a d'ailleurs à l'esprit les mots de Eduardo Souto de Moura lorsqu'il lui fut demandé d'écrire un essai pour un recueil en mémoire de Luigi Snozzi. II paraphrasa Maurice Merleau-Ponty: "L'artiste n'a qu'un moyen de représenter l'œuvre sur laquelle il travaille. C'est la faire ${ }^{32}$. " D'une certaine manière, il entendait proclamer le divorce existant entre le verbe et le projet architectural. Mais en s'inspirant de la phénoménologie, il soulignait malgré tout que le propre d'une réflexion sur une œuvre était précisément de «revenir sur l'œuvre même ». Mais l'œuvre est-elle un objet ou un sujet?

Dans une sorte d'hyperbole sensible, l'architecte (parfois à son corps défendant) qui fait un projet, apporte de la connaissance. L'architecture n'est pas simplement la réponse opportune à une question programmatique, elle suscite des questionnements qui correspondent à son déploiement dans l'univers du sens et non simplement du sensible. Or, la grande difficulté du doctorat en architecture consiste à inventer les règles de cette poïétique qui trouverait dans la production architecturale les fondements de sa propre mise en perspective. Cela peut, sans doute, s'opérer dans l'hyper-contextualisme ${ }^{33}$ des pensées génériques contemporaines, mais demanderait à être confronté à l'échelle du temps et de l'histoire pour s'approcher de cette quête de vérité, réclamée par les sophistes mais impossible à accepter dans l'espace (non fini) de l'architecture. II suffit, pour en prendre conscience, de déplacer les curseurs des discours contemporains pour le mesurer.

Ainsi par exemple, nombreux furent les architectes (et les chercheurs) qui voulurent imposer un certain formalisme lexicographique dans l'étude et la composition des façades. L'actuelle religion au du culte énergétique a d'ailleurs encore accéléré ce processus. On vit même élever au rang de recherche architecturale des processus de composition de façades thermiquement
18. À la différence de 306090 qui se veut à destination de jeunes architectes chercheurs, Pamphlet Architecture assume la place de la recherche dans la pensée contemporaine de l'architecture. Sont présents parmi les auteurs Perry Kulper, Nat Chard, ainsi que quelques figures majeures de la création architecturale contemporaine.

19. Publiés dans leur nouvelle version depuis 1981 par l'Architectural Association School of Architecture de Londres.
20. Développée au sein de la School of Architecture + Planning du MIT, SA + P Press propose une exploration des thèmes et des postures architecturales contemporaines.

21. Laboratory for Integrative Architecture, Technische Universität Berlin, professeur Finn Geipel (carrot.a.tu-berlin.de).

22. Nous pensons par exemple à Marnes (Ensa de la Ville et des Territoires, Marne-la-
Vallée), aux Cahiers thématiques (Ensap de Lille), etc., auxquelles on pourrait associer les revues critiques d'architecture dont les relations avec les enseignants chercheurs des Ensa sont immédiates: Criticat, Le Visiteur, etc., et bien évidemment la revue dans laquelle prend place cet article.

23. Cela dessine la "matérialité » du discours et sa formidable force d'exclusion, voir Michel Foucault, L'Ordre du discours, Paris, Gallimard, 1971, p. 47-48. 
exceptionnelles mais d'une monstruosité apocalyptique, au prétexte que ces façades se posaient au devant d'architectures. Une réponse technique à une question technique n'est pas de l'architecture: elle demeure une réponse technique, rien de plus ${ }^{34}$. Dans le monde de l'architecture, une façade ne s'appelle pas une enveloppe. Elle n'est pas le masque d'un édifice. Elle demeure une peau qui participe pleinement du sens de l'œuvre architecturale. Or, comme chacun sait, une peau est une surface de contact, absolument pas une carapace à la pauvreté. C'est ce type d'évidence qui distingue les manières de penser d'un architecte et qui, par conséquent, permet de dissocier le doctorat en architecture du doctorat en thermique, en ingénierie ou en génie civil. S'engager dans un doctorat en architecture signifie ne jamais faire le deuil de son métier, de sa langue et de tout ce qui a construit la posture de l'architecte.

\section{Un procès au réel}

Tout programme architectural est une abstraction de l'espace, une abstraction du réel. Norman Foster disait même que pour l'édification du Carré d'art de Nîmes, il avait «inventé » le lieu. En un certain sens, il avait raison. Toute architecture transforme, pervertit ou parfois même révèle le territoire de son implantation ${ }^{35}$. Faut-il l'admettre et s'affirmer dans la démiurgie de la création, ou faut-il, au contraire être retors avec le lieu et imaginer ces "topologies rusées " dont parlait Henri Gaudin ${ }^{36}$ ? La réponse est impossible, mais quelle que soit la réponse architecturale à un programme, le territoire de l'architecte escamotera le réel et en prononcera même le procès.
Un parallèle pourrait facilement s'établir avec le monde de la recherche en musicologie, ou plus précisément encore avec la recherche en interprétation musicale. C'est dans l'appropriation personnelle de la pensée d'autrui, en l'occurrence d'un compositeur, que se construit l'art de l'interprétation. Personne ne s'insurge face à l'interprétation par Glenn Gould des Variations Goldberg. Pourtant entre I'enregistrement de 1955 et la célèbre intégrale de 1981, les Variations gagnèrent presque onze minutes. Jean-Sébastien Bach y gagna en profondeur, en retenue, en subtilité aussi. Glenn Gould n'était pas simplement l'interprète de Bach, il était un musicien ${ }^{37}$. Être musicien c'est puiser dans une œuvre - qui est en quelque sorte la réponse écrite à un programme harmonique - sa qualité transcendante.

Sans même forcer le trait, être architecte participe d'une même dynamique. De même qu'il existe un doctorat en interprétation musicale, il devrait exister dans le doctorat en architecture une part de cette quête personnelle vers la transcendance. La parfaite maîtrise des instruments et des règles de composition permet au musicien de dépasser les simples savoir-faire pour s'approcher de l'entière dimension de l'œuvre. En architecture, ce que l'on nomme "le projet " relève de la même démarche. II s'agit de posséder la complétude des outils pour pouvoir totalement s'en libérer. La formule souvent entendue: "cette personne parle comme un architecte ", au-delà de son caractère de défiance, signifie bien que l'architecte manie une langue (il faudrait plutôt dire un langage) qui définit sa personnalité. Un doctorat en architecture devrait donc être la quintessence de cette langue.
24. Voir notamment Dominique Rouillard (dir.), L'infraville/Futur des infrastructures, Paris, Archibooks, 2011.

25. Cyrille Simonnet, Philippe Potié (dir.), "Culture constructive », Cahiers de la Recherche architecturale, $n^{\circ} 29$, Marseille, Parenthèses, 1992

26. Andrea Branzi, Nouvelles de la métropole froide: design et seconde modernité, Paris, Éditions du Centre Pompidou, 1992.
27. Nathalie Lancret, « Représentation des espaces urbains en mutation et imaginaire métropolitain », dans Frédéric de Coninck et José-Frédéric Deroubaix (dir.), Transformations des horizons urbains. Savoirs, imaginaires et usages en conflit, Paris, L'CFil d'or, 2012, p. 287-291.

28. Bénédicte Grosjean, «Paola Viganò : à la recherche de la métropole horizontale ", Criticat, $n^{\circ}$ 10, septembre 2012, p 16-35.
29. Sébastien Marot, Sub-urbanism and the Art of Memory, Londres, AA Paperback Publications, 2003

30. On peut même s'interroger pour savoir si les acquis des exigences de rationalisation américaine lors de la Seconde Guerre mondiale n'ont pas accéléré certains processus d'uniformisation et de standardisation rapides. Voir: Jean-Louis Cohen, Architecture en uniforme. Projeter et construire pour la Seconde Guerre 
Les comparaisons avec les travaux menés dans d'autres disciplines universitaires sont, en ce sens, particulièrement stimulantes. En suivant l'évolution du doctorat en activités physiques et sportives, ou du doctorat en arts plastiques, on assiste à l'émergence de ce que l'on pourrait nommer la maturité disciplinaire. Dans les deux cas, la volonté de la part des chercheurs d'inventer la poïétique de leur discipline a permis l'élaboration d'une identité de la recherche. Le postulat de la démarche scientifique n'était pas de cloner des processus doctoraux existants, mais bien de construire à la fois les matériaux et les outils de leur recherche. Un doctorat en arts plastiques est à la fois création individuelle et interrogation réflexive sur le concept même de création. Un doctorat en STAPS est à la fois une étude liant le sport à l'être, mais en même temps une interrogation, là encore réflexive, sur le sport comme discipline.

L'architecture, et l'enseignement de l'architecture, étant restés si longtemps sous la dépendance d'autres disciplines, les chercheurs en architecture ont pensé qu'ils devaient plagier des méthodologies étrangères pour circonscrire le cadre de leur réflexion. Cette timidité dans l'affirmation disciplinaire est préjudiciable à l'architecture et peut même être considérée comme un handicap conceptuel. L'évolution actuelle du doctorat en architecture porte l'espérance d'une modification. Cela signifie des contraintes et surtout l'obligation de la part des chercheurs de revendiquer une posture scientifique " autre ». L'architecture est un jeu d'acteurs alors que la recherche est une entreprise solitaire dans le monde des humanités. II devient par conséquent indispensable pour la France qu'une génération de chercheurs engagés dans cette discipline soit capable de construire les outils d'une interrogation nouvelle pour façonner une méthodologie propre à la recherche architecturale.

La chose n'est pas aisée. Car le risque est grand de croire que lorsque l'architecte fait un projet, il fait une recherche. Non, il exerce simplement son métier. La recherche en architecture, c'est précisément dépasser le métier pour, peu à peu, interroger la fabrication de cette pensée complexe. Croirait-on par exemple qu'un avocat préparant sa plaidoirie, avec force références au droit et à l'histoire du droit, à la jurisprudence, à l'histoire personnelle d'un prévenu, avec un savoir-faire rhétorique dont la phraséologie et les effets de manche constituent la mise en scène, entamât une recherche doctorale? Absolument pas. On reconnaîtrait en lui, un professionnel usant à la perfection des outils de sa profession. Dans le monde de l'architecture, il en est de même. C'est pourquoi le doctorat en architecture n'est pas un doctorat professionnel, ni même un doctorat d'exercice tel qu'il se rencontre en médecine. II est cette réévaluation du "faire " dans une posture critique propre à tout processus de conception. Réévaluer le «faire » n'est pas faire l'apologie d'une technicité d'agence, mais bien envoyer un défi au réel. $\mathrm{Ne}$ jamais se satisfaire de l'attendu d'une remarque, mais demeurer dans l'attente de cette remarque. En ce sens, lorsque les architectes dits "postmodernes" ont pillé les livres d'images pour plaquer sur des constructions insipides des pastiches baroques ou néoclassiques, ou lorsque les architectes néomodernes collent à coups de photocopies des signes corbuséens, ou tessinois, sur leurs cubes de béton, ils pervertissent la logique même de «la pensée de projet ${ }^{38}$.
mondiale/Architecture in Uniform. Designing and Building for the Second World War, Paris, Hazan, 2011, p. 11-29.

31. Voir la mise au point par Martine Bouchier, 10 clefs pour s'ouvrir à I'architecture, Paris, Archibooks, 2009, ainsi que le voyage "libre » dans le vocabulaire d'un art partagé : Florence Lipsky, Pascal Rollet, Les 101 mots de l'architecture à I'usage de tous, Paris, Archibooks, 2009
32. Eduardo Souto de Moura, "Ceci n'est pas un texte ", dans Pierre-Alain de Croset, Luigi Snozzi (dir.), Pour une école de Tendance. Mélanges offerts à Luigi Snozzi, Lausanne, Presses polytechniques et universitaires romandes, 1999, p. 93-99. II reprend ici la comparaison établie par Merleau-Ponty encore prononciation et représentation: «[...] Comme l'artiste n'a qu'un moyen de se représenter l'œuvre à laquelle il travaille: il faut qu'il la fasse. » Maurice Merleau-Ponty, Phénoménologie de la perception, Paris, Gallimard, 1989, p. 221

33. Un axiome à la mode voudrait en effet que la compréhension " complète » du contexte permette d'inventer l'architecture parfaite (c'est-à-dire que l'idea eut laissé la place à l'efficacité...).

34. Il faudrait reprendre ce petit texte ancien, mais si plein de belles choses: Lisa Heschong, Thermal Delight in Architecture, Cambridge (MA), MIT Press, 1979 (trad. 
L'architecture s'écrit dans sa matérialité. Quelle que soit son échelle, sa fonction ou son implantation, quelle que soit son usage ou son expression plastique, une architecture s'installe dans I'horizon des villes et des territoires par sa présence physique. Dans la lente et difficile mise en œuvre d'un projet qui concentre la complexité d'un dessein et l'ascèse d'un chantier, le réel englue. Le quotidien de l'agence, comme le rythme des réunions entre corps de métier sur le chantier, usent et condamne à l'efficacité. Depuis des siècles il en va ainsi et tous les mémoires rédigés par des architectes reprennent la même antienne du labeur et de l'épreuve. Mais depuis des siècles aussi, les architectes ont éprouvé le besoin de se poser à l'écart la pénibilité d'un métier pour interroger les principes mêmes de leur création. II ne s'agit pas là d'une littérature architecturale normalisée, puisqu'elle associe plutôt les ouvrages techniques, utiles au "métier», et les analyses théoriques sur les œuvres d'autrui. Non, il s'agit plutôt d'une tentative pour circonscrire le faire de son propre travail dans le surgissement des savoirs qui constituent un individu. II est peut-être difficile de décerner à ces textes le titre de recherche, au sens académique et actuel du terme. Ces textes constituent cependant le fondement de l'écriture architecturale. Lorsqu'on les rattache à la littérature architecturale, ils sont indexés sous l'étiquette d'essais. Pourtant, ils constituent certainement le meilleur exercice de poïétique que l'on puisse trouver, et participent pleinement de la définition du périmètre disciplinaire de l'architecture ${ }^{39}$. Leur particularité est de ne pas se complaire dans une logorrhée descriptive de l'existant, pour préférer les questionnements et les interrogations.
Cela signifie quitter le confort du matérialisme technicien pour pénétrer le monde de la pensée.

Être soucieux uniquement du réel, et faire de la réalité une religion, amèneraient les architectes à disparaître derrière des réponses opportunes, réglementairement admissibles et humainement obscènes. Paul Valéry résuma bien se danger qui pointait sous la recherche de l'évidence réaliste. II écrivit: "L'excès de réalité, cela s'appelle aussi la pornographie ${ }^{40}$. »

L'architecte est donc condamné à faire le procès du réel, à rejeter avec violence cette gangue qui ensevelit la pensée sous prétexte d'efficacité économique, technique et réglementaire. Toute la culture de projet, telle que nous l'évoquions plus haut, est à l'épreuve dans cette posture intellectuelle. Refuser le réel, tel qu'il nous est donné par les médias, par les maîtres penseurs que sont devenus les techniciens de la ville et les fonctionnaires du goût, telle est la dignité d'une discipline. Un réel élevé en dogme de vérité dénoncerait les penseurs comme des songes creux. Ainsi que le rappelait Nietzsche: «Nous avons l'art afin de ne pas mourir de la vérité ${ }^{41} \ldots »$

Un procès n'est pas une condamnation, mais au contraire le parcours permettant de circonscrire une idée pour la faire nôtre, la comprendre, la critiquer, l'approprier et la transformer dans une perception qui est notre propre téléologie. Les travaux, recherches, prophéties, autour de la question des posthumains sont à cet égard passionnants.

La question n'est plus Où vivrons-nous demain ${ }^{42}$, dont l'angélisme naïf nous fait aujourd'hui sourire, mais bien quel espoir est encore donné à l'homme de vivre demain? L’humanité augmentée est déjà notre présent. Le « déjà fr. Architecture et volupté thermique, Marseille, Parenthèses, 1992).

35. Danièle Voldman, «Les traceurs de villes ont-ils un discours de démiurges? ", dans Loïc Vadelorge (dir.), Gouverner les villes nouvelles. Le rôle de l'État et des collectivités locales (1960-2005), Paris, Le Manuscrit, coll. « Manuscrit Université », 2005, p. 51-66.

36. Henri Gaudin, La cabane et le labyrinthe, Bruxelles, Mardaga, 1983, p. 67.
37. Peter Ostwald, Glenn Gould. The Ecstasy and Tragedy of Genius, New York, W. W. Norton \& Company, 1998, p. 51-72.

38. D'une certaine manière, la pensée postmoderne revendique, par-delà l'esthétisme, la possibilité de tout accepter comme signifiant sans interroger le signifié de l'œuvre. Voir : Richard Shusterman, "Postmodern Aestheticism : a New Moral Philosophy? ", Theory, Culture an Society n०5, 1988, p. 337-355.
39. II est d'ailleurs assez singulier de constater que les anthologies sur I'architecture furent composées jusqu'au milieu $\mathrm{du} x \mathrm{x}^{\mathrm{e}}$ siècle à partir de textes écrits par des architectes praticiens, puis, à partir des années 1970, à partir de textes de critiques (parfois architectes) entretenant avec le métier une relation plus distanciée. $C^{\prime}$ 'est tout au moins ce que semblait souligner, pour l'architecture espagnole, Antonio Fernández Alba, La crisis de la arquitectura española 1939-1972, Madrid, 
là » des virtualités connectées et des êtres médicalement assistés (par greffe, prothèses, implants...) est l'évidence de notre condition. Le réel est mutant. L'avenir incertain et pourtant déjà inventé. La place de l'architecture dans le changement de paradigme qui situe l'homme face à une cyber-conscience n'est jamais interrogée. Les techno-prophètes du dispositif posthumain fabriquent des imaginaires architecturaux, urbains et paysagers dont le futurisme a quelque chose de désuet. Qui donc a été capable d'inventer la forme d'une révolution incarnée? Personne, or cet enjeu, déterminant pour la condition même de la recherche en architecture, ne peut être laissé à des astucieux techniciens de la domotique ou à des illustrateurs de jeux vidéo.

L'architecture va au-delà de la forme, et tout doctorat en architecture ne peut passer outre cette évidence. La seule évidence de la démarche architecturale est I'homme. Un homme fragile, inconséquent, profondément sensible face à la quête d'un bonheur passager et indispensable. La condition postmoderne qui est une sorte d'apothéose du narcissisme individuel fabrique un nouveau rapport à l'architecture et à son devenir. L'architecture représente une tentative pour produire le durable (qui n'est ici pas un label pour thermicien) d'un monde qui change de paradigme et a troqué les rêves de I'humanisme pour les chimères de l'égoïsme posthumain. Plus que jamais, l'architecte qui a été fabriqué depuis des siècles dans la générosité de "cette main ouverte pour donner », comme disait Le Corbusier, se doit d'interroger la plénitude de sa propre discipline.

Avec cette double voie d'un doctorat en architecture ouvert soit sur des approches disciplinaires variées, tel qu'il se fait actuellement au sein des laboratoires des écoles d'architecture, soit sur des approches davantage orientées vers les logiques de projet, peut se dessiner l'avenir de la recherche en architecture. Ces deux options ne sont pas incompatibles et la production scientifique de ces quarante dernières années en est le témoignage. Mais une ouverture réflexive aux travaux architecturaux contemporains semble indispensable.

$\mathrm{Ce}$ n'est qu'avec cette transformation que l'architecture parviendra à s'affirmer comme discipline. Le particularisme français qui situe l'enseignement d'une discipline dans un ministère (la Culture) qui n'a aucune tradition universitaire, ni aucune tradition scientifique (au sens épistémologique du terme) doit aussi être I'objet de réflexions. II y a là une chance et une difficulté. Une chance, car l'architecture n'est pas contrainte par le poids d'héritages disciplinaires issus de la longue tradition universitaire. Une difficulté, car l'architecture doit construire son autonomie disciplinaire dans la perspective d'échapper aux regards circonspects d'autres disciplinaires vénérables. Cela aurait dû se faire depuis longtemps. Mais cette marginalisation dans l'enseignement supérieur devrait assez vite disparaître si le doctorat en architecture n'est plus un unique vocable réglementaire, mais l'expression réfléchie d'une discipline majeure, inscrite (comme dans le reste du monde) au cœur des universités ${ }^{43}$. En continuant à défendre l'idée que le doctorat en architecture est un procès au réel et non un asservissement aux exigences de savoirs contingents, la discipline architecture s'affirmera de plus en plus dans sa confluence entre sciences et techniques, entre humanités et sciences sociales.
Cuadernos para el Diálogo, Edicusa, 1972, p. 119-128.

40. Paul Valéry, Mon Faust, Paris, Gallimard, 1946, p. 28.

41. Friedrich Nietzsche, Fragments posthumes, 16 [40], §6, dans Fuvres philosophiques complètes (Giorgio Colli, Maurizio Montinari, dir.), trad. fr., Paris, Gallimard, 1698-1997 [OK pour ces dates?] vol. XIV, p. 250.
42. Michel Ragon, Où vivrons-nous demain? Paris, Robert Laffont, 1963. Ouvrage auquel il serait tentant de confronter: Béatrice Mariolle, Philippe Villien, Subagglo 2030, Grand Paris hors les murs, Paris, Éditions Recherches, 2011, qui introduit le concept de "subagglo " dans l'analyse des grandes aires agglo-métropolitaines et s'intéresse davantage aux territoires habités qu'à I'esthétisation futuriste de modes de vie imaginaires.
43. II faudrait presque dire " au centre», tant les universités anglo-saxonnes, germaniques, méditerranéennes, asiatiques ou scandinaves ont élaboré leur campus et leurs strates disciplinaires à partir des facultés d'architecture. 